\title{
HEMATOMA INTRAMURAL DUODENAL COMO COMPLICAC̣ÃO DE TERAPIA ANTICOAGULANTE COM WARFARIN: RELATO DE CASO E REVISÃO DA LITERATURA*
}

\author{
Juliano Faria ${ }^{1}$, Roberta Pessoa ${ }^{2}$, Marcelo Hudson ${ }^{2}$, Sílvio Vitoi $^{2}$, Ovídio Villela $^{3}$, José Torres ${ }^{3}$, \\ Mara Delgado Paula ${ }^{4}$, Aloísio Bemvindo ${ }^{5}$
}

\begin{abstract}
Resumo Relatamos o caso de uma paciente em terapia anticoagulante oral com Warfarin, apresentando obstrução intestinal aguda. A tomografia computadorizada revelou hematoma intramural duodenal. 0 tratamento baseou-se na correção das provas de coagulação e medidas expectantes. Este caso ilustra o valor da tomografia computadorizada e da abordagem conservadora nos pacientes em terapia anticoagulante com obstrução aguda do intestino delgado.

Unitermos: Hematoma duodenal; Warfarin; Anticoagulantes.
\end{abstract}

\begin{abstract}
Intramural duodenal hematoma as a complication of therapy with Warfarin: a case report and literature review.

We report a case of a patient receiving chronic oral anticoagulant therapy with Warfarin who presented with acute intestinal obstruction. Computed tomography showed intramural duodenal hematoma. Treatment was conservative with correction of the coagulation parameters and observation. This case exemplifies the usefulness of conservative therapy and computed tomography in patients with acute small bowel obstruction receiving anticoagulant therapy.

Key words: Duodenal hematoma; Warfarin; Anticoagulant therapy.
\end{abstract}

\section{INTRODUÇÃO}

A dor abdominal aguda em pacientes em terapia anticoagulante oral se apresenta como um problema diagnóstico e um desafio terapêutico. Os hematomas de parede intestinal foram alvo de raras publicações na literatura mundial — com aproximadamente 120 casos descritos - , tendo como fator etiológico a terapia anticoagulante ${ }^{(1-13)}$. A maior série descrita foi de 13 pacientes, indicando que nenhuma instituição possui experiência acumulada na condução do problema ${ }^{(\mathbf{1})}$. Os achados da ul-

* Trabalho realizado no Serviço de Diagnóstico por Imagem do Hospital Márcio Cunha, Fundação São Francisco Xavier, Ipatinga, MG.

1. Médico Pós-graduando do Departamento de Diagnóstico por Imagem da Universidade Federal de São Paulo/Escola Paulista de Medicina (Unifesp/EPM).

2. Médicos Residentes do Serviço de Diagnóstico por Imagem do Hospital Márcio Cunha.

3. Médicos Radiologistas do Serviço de Diagnóstico por Imagem do Hospital Márcio Cunha, Membros Titulares do Colégio Brasileiro de Radiologia e Diagnóstico por Imagem.

4. Médica Radiologista do Serviço de Diagnóstico por Imagem do Hospital Márcio Cunha.

5. Médico Infectologista, Coordenador do Serviço de Terapia Intensiva do Hospital Márcio Cunha.

Endereço para correspondência: Dr. Juliano Faria. Rua Itapiru, 598, ap. 125, Saúde. São Paulo, SP, 04143-010. E-mail: drjuliano@uol.com.br

Recebido para publicação em 22/10/2003. Aceito, após re visão, em 5/1/2004. tra-sonografia abdominal e da tomografia computadorizada também foram descritos em poucos relatos ${ }^{(1-7)}$.

Neste artigo descrevemos um caso e discutimos a etiologia, sinais clínicos, diagnóstico e manejo do hematoma intramural.

\section{RELATO DO CASO}

Paciente do sexo feminino, 64 anos de idade, leucodérmica, portadora de cardiopatia chagásica com fibrilação atrial persistente, em terapia anticoagulante oral com Warfarin, há dois meses, admitida no Hospital Márcio Cunha, apresentando extensas manchas equimóticas por todo o corpo, especialmente nos membros e face ( $\mathrm{Fi}-$ gura 1), além de dor abdominal difusa há 48 horas associada a vômitos.

A paciente evoluiu com quadro de obstrução intestinal aguda. Dados laboratoriais à internação hospitalar incluíam tempo de tromboplastina parcial de 126 segundos (controle: 30 segundos), tempo de protrombina superior a 127 segundos (controle: 11 segundos), atividade de protrombina inferior a $5 \%$ (controle: $100 \%$ ), índice de reticulócitos superior a 16,00, hematócrito de 30,3\%, hemoglobina de 10,2 g/dl, plaquetas de $315.000 / \mathrm{mm}^{3}$, leucócitos de $16.200 / \mathrm{mm}^{3}$.

A radiografia simples de abdome evidenciou sinais de obstrução intestinal. Exame ultra-sonográfico do abdome foi realizado, visualizando-se segmento de alça de intestino delgado com parede bastante espessada, hipoecogênica, além do estômago e alças proximais distendidos e preenchidos por líquido (Figura 2). O diagnóstico não invasivo foi possível realizando-se tomografia computadorizada de abdome, que revelou hematoma intramural duodenal obstruindo completamente o trânsito intestinal (Figura 3).

O tratamento baseou-se na suspensão do anticoagulante, correção das provas de coagulação e medidas expectantes (jejum, sonda nasogástrica, vitamina $\mathrm{K}$ e suporte hidroeletrolítico), obtendo-se resultado positivo em dez dias. No dia anterior à alta hospitalar, foi realizado exame tomográfico de controle, evidenciando resolução do hematoma (Figura 4).

\section{DISCUSSÃO}

O Warfarin é um anticoagulante cumarínico que interfere com a carboxilação 


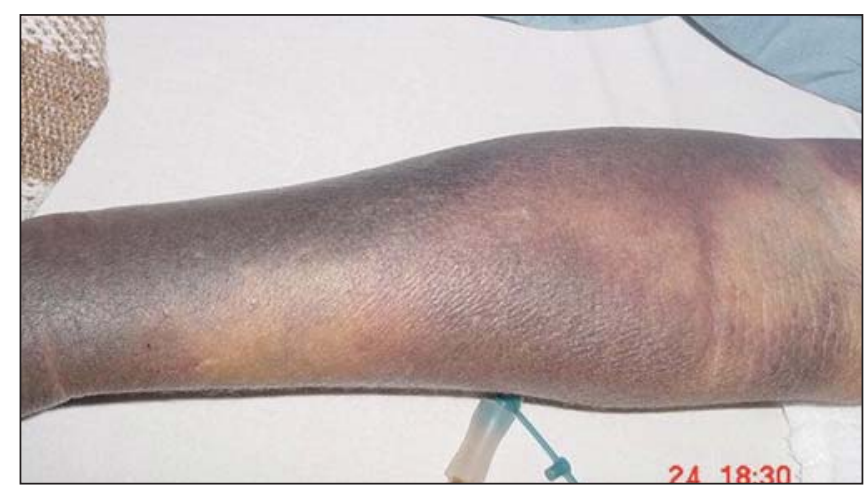

A

Figura 1. A: Foto do antebraço da paciente à admissão evidenciando extensa equimose. B: Foto da paciente no dia da alta hospitalar, com melhora expressiva da equimose.

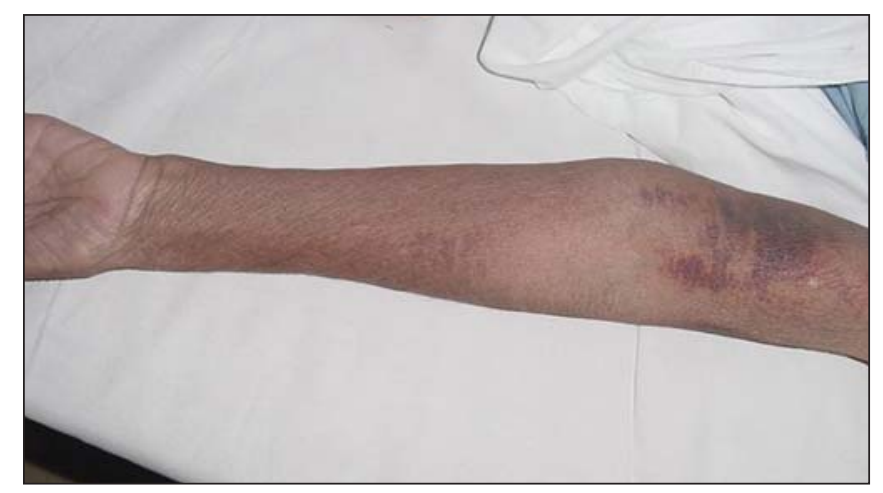

B

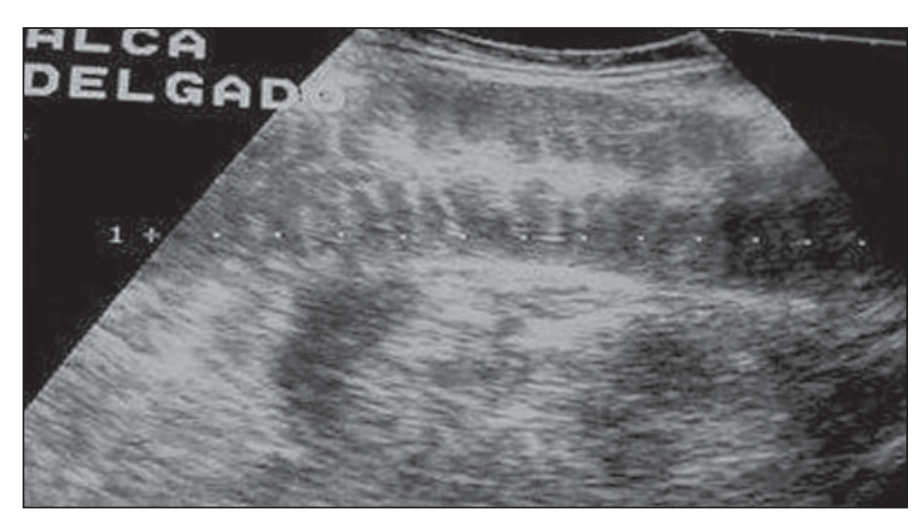

Figura 2. Exame ultra-sonográfico evidenciando alça duodenal hipoecóica, espessada e com redução da luz.

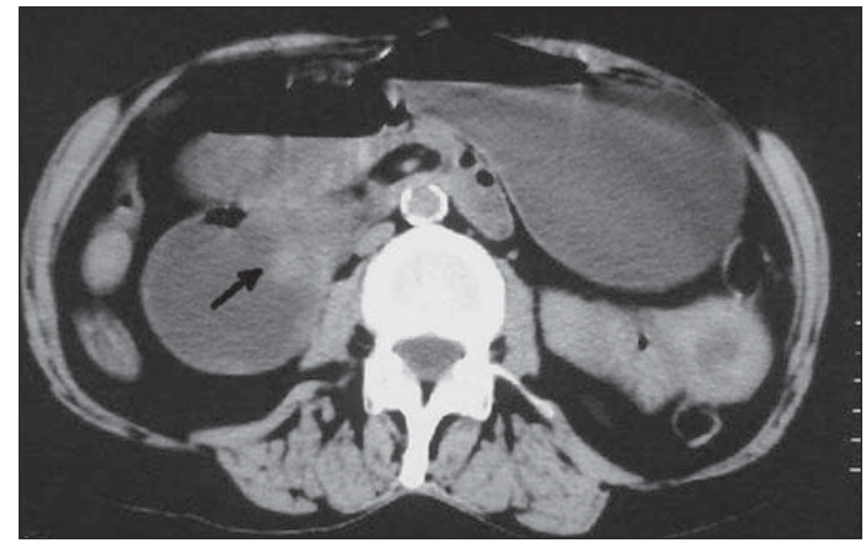

Figura 3. Exame de tomografia computadorizada do abdome evidenciando distensão do estômago e da primeira e segunda porções do duodeno, e hiperdensidade parietal duodenal, com obstrução da luz intestinal (seta).

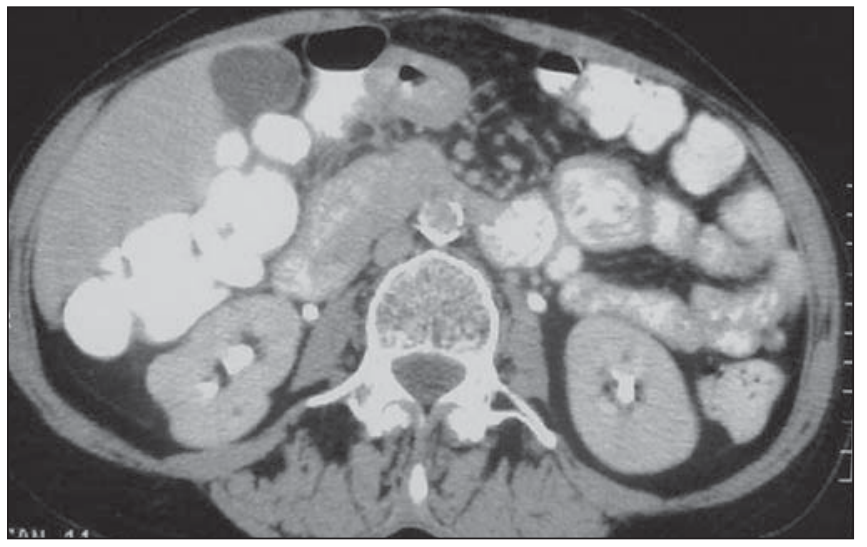

Figura 4. Controle tomográfico evidenciando redução da hiperdensidade parietal duodenal e trânsito duodenal livre. hepática vitamina K-dependente dos fatores II, VII, IX e X. É utilizado em pacientes com tromboembolismo pulmonar, trombose venosa profunda, próteses valvares, ou fibrilação atrial persistente ${ }^{(\mathbf{1 4})}$. O risco de sangramento maior em pacientes em terapia anticoagulante oral é da ordem de $3 \%$ ao ano, sendo que a idade acima de 75 anos é o fator de risco mais importante ${ }^{(\mathbf{1 5})}$. O sangramento intramural intestinal não é complicação comum da terapia anticoagulante, tendo sido descrito pela primeira vez em 1952, causando obstrução intestinal e hemoperitônio ${ }^{(\mathbf{1 1})}$, entretanto, atualmente, tem sido relatado com certa frequiência. A maior parte dos casos relatados na literatura ocorreu em homens com cerca de 60 anos de idade. A anticoagulação pelo War- farin é a causa mais comum de hematoma intramural espontâneo do intestino delgado. Outros fatores de risco incluem hemofilia, púrpura trombocitopênica idiopática, leucemia, linfoma, mieloma, quimioterapia, vasculite, pancreatite e carcinoma pancreático $^{(\mathbf{1 , 2})}$. Os locais de acometimento mais frequientes são o duodeno e o jejuno proximal, sendo que o hematoma único 
ocorre mais frequentemente que os hematomas múltiplos ${ }^{(\mathbf{1 , 4 - 6})}$. A natureza altamente vascular do duodeno pode ser um fator importante na ocorrência de hematomas nesta região ${ }^{(4,6)}$.

Os sintomas mais comuns desses pacientes são náuseas, vômitos e dor abdominal $^{(\mathbf{6})}$. Os testes laboratoriais e achados radiológicos convencionais são inespecíficos, mas quando associados nos pacientes em terapia com anticoagulantes orais, a suspeita torna-se necessária ${ }^{(4)}$. Estima-se que um sangramento intramural com volume de cerca de $30 \mathrm{ml}$ possa produzir obstrução intestinal $^{(\mathbf{1 2})}$. A grande questão nestes pacientes é que eles se apresentam ao cirurgião com quadro de abdome agudo, tipicamente epigastralgia e vômitos contendo bile, podendo ocorrer anormalidades laboratoriais como leucocitose, aumento da bilirrubina sérica e da amilase (por obstrução dos fluxos biliar e pancreático), complicando o diagnóstico ${ }^{(3-6)}$. Caso não seja feito o diagnóstico correto em tempo hábil, o paciente pode ser levado inadvertidamente à laparotomia exploradora, agravando seu quadro clínico. Um fator importante a ser lembrado é o achado de que cerca de $84 \%$ dos casos de abdome agudo nos pacientes em terapia anticoagulante se deve a hemorragia intramural. Outro fator é a coexistência dos hematomas com outras afecções abdominais, como infarto mesentérico e apendicite aguda ${ }^{(7)}$.

Em relação aos métodos de imagem, a tomografia computadorizada é o exame mais indicado para o diagnóstico correto e precoce dos hematomas intramurais, que se apresentam como massas de alta atenuação, homogêneas, associadas com alterações obstrutivas ${ }^{(\mathbf{1 - 6 , 8 , 9 )}}$. Os sinais tomográficos incluem espessamento circunferencial da parede intestinal, hiperdensidade intramural, estreitamento luminal e obstrução intestinal, e são visualizados principalmente nos dez primeiros dias ${ }^{(\mathbf{1})}$. A tomografia pode auxiliar também na identificação de complicações do hematoma intramural, como os abscessos e perfurações ${ }^{(3)}$. Os achados do exame ultra-sonográfico consistem de submucosa espessada e ecogênica, mas esta anormalidade não é específica para hematomas do trato intestinal. O exame ultra-sonográfico também pode evidenciar a extensão do processo. Nas imagens da ressonância magnética ponderadas em T2 foi descrito o "sinal do anel", que identifica o hematoma duodenal, auxiliando na distinção entre outras massas e coleções abdominais. Como é um exame de alto custo e baixa disponibilidade, é reservado para casos em que outras técnicas falham em estabelecer o diagnóstico ${ }^{(\mathbf{9})}$.

O diagnóstico, tendo sido estabelecido, o tratamento de escolha é conservador ${ }^{(1-9)}$. Vitamina K, suporte hidroeletrolítico, jejum e sonda nasogástrica são as condutas básicas. O quadro geralmente tem sua resolução em até três semanas. Não ocorrendo em até dois meses, o diagnóstico deveria ser repensado pelo radiologista. Este caso ilustra o valor da tomografia computadorizada e da abordagem conservadora nos pacientes em terapia anticoagulante que se apresentam com obstrução aguda do intestino delgado.

\section{REFERÊNCIAS}

1. Abbas MA, Collins JM, Olden KW. Spontaneous intramural small-bowel hematoma: imaging findings and outcome. AJR 2002;179:1389-94.

2. Lane MJ, Katz DS, Mindelzun RE, Jeffrey RB Jr. Spontaneous intramural small bowel haemorrhage: importance of non-contrast CT. Clin Radiol 1997; 52:378-80.

3. Farhoud S, Stephani SM, Bromberg SH. Pancreatite aguda devida a hematoma intramural do duodeno por uso de anticoagulante. Arq Gastroenterol 2001;38:53-6.

4. Avent ML, Canaday BR, Sawyer WT. Warfarin-induced intramural hematoma of the small intestine. Clin Pharm 1992;11:632-5.

5. Gutstein DE, Rosenberg SJ. Nontraumatic intramural hematoma of the duodenum complicating warfarin therapy. Mt Sinai J Med 1997;64:339-41.

6. Shah P, Kraklow W, Lamb G. Unusual complication of coumadin toxicity. Wis Med J 1994;93: 212-4.

7. Euhus DM, Hiatt, J.R. Management of the acute abdomen complicating oral anticoagulation therapy. Am Surg 1990;56:581-6.

8. Hughes JJ, Brogdon BG. Computed tomography of duodenal hematoma. J Comput Tomogr 1986;10: 231-6.

9. Hahn PF, Stark DD, Vici LG, Ferrucci JT Jr. Duodenal hematoma: the ring sign in MR imaging. Radiology 1986;159:379-82.

10. Jones WR, Hardin WJ, Davis JT, Hardy JD. Intramural hematoma of the duodenum: a review of literature and case report. Ann Surg 1971;173:534 44.

11. Berman H, Mainella FS. Toxic results of anticoagulant therapy. N Y State J Med 1952;52:725-7.

12. Hughes CE 3rd, Conn J Jr, Sherman JO. Intramural hematoma of the gastrointestinal tract. Am J Surg 1977;133:276-9.

13. Cocks JR. Anticoagulants and the acute abdomen Med J Aust 1970;1:1138-41.

14. Hirsh J. Oral anticoagulant drugs. N Engl J Med 1991;324:1865-75.

15. Launbjerg J, Egeblad H, Heaf J, Nielsen NH, Fugleholm AM, Ladefoged K. Bleeding complications to oral anticoagulant therapy: multivariate analysis of a 1010 treatment years in 551 outpatients. J Intern Med 1991;229:351-5. 